\title{
MICROWAVE CONTINUUM EMISSION AND DENSE GAS TRACERS IN NGC 3627: COMBINING JANSKY VLA AND ALMA OBSERVATIONS
}

\author{
Eric J. Murphy ${ }^{1}$, Dillon Dong ${ }^{2}$, Adam K. Leroy ${ }^{3}$, Emmanuel Momjian ${ }^{4}$, James J. Condon $^{5}$, George Helou ${ }^{6}$, \\ David S. Meier ${ }^{4,7}$, Jürgen OtT ${ }^{4}$, Eva Schinnerer ${ }^{8}$, and Jean L. Turner ${ }^{9}$ \\ ${ }^{1}$ Infrared Processing and Analysis Center, California Institute of Technology, MC 314-6, Pasadena, CA 91125, USA; emurphy@ipac.caltech.edu \\ ${ }^{2}$ Department of Physics and Astronomy, Pomona College, Claremont, CA 91711, USA \\ ${ }^{3}$ The Ohio State University, 140 W18th Street, Columbus, OH 43210, USA \\ ${ }^{4}$ National Radio Astronomy Observatory, P.O. Box O, 1003 Lopezville Road, Socorro, NM 87801, USA \\ ${ }^{5}$ National Radio Astronomy Observatory, 520 Edgemont Road, Charlottesville, VA 22903, USA \\ ${ }^{6}$ California Institute of Technology, MC 100-22, Pasadena, CA 91125, USA \\ ${ }^{7}$ New Mexico Institute of Mining \& Technology, 801 Leroy Place, Socorro, NM 87801, USA \\ ${ }^{8}$ Max Planck Institut für Astronomie, Königstuhl 17, D-69117 Heidelberg, Germany \\ ${ }^{9}$ Department of Physics and Astronomy, UCLA, Los Angeles, CA 90095, USA \\ Received 2015 May 24; accepted 2015 August 21; published 2015 November 5
}

\begin{abstract}
We present Karl G. Jansky Very Large Array Ka band (33 GHz) and Atacama Large Millimeter Array (ALMA) Band $3(94.5 \mathrm{GHz})$ continuum images covering the nucleus and two extranuclear star-forming regions within the nearby galaxy NGC 3627 (M 66), observed as part of the Star Formation in Radio Survey. Both images achieve an angular resolution of $\lesssim 2^{\prime \prime}$, allowing us to map radio spectral indices and estimate thermal radio fractions at a linear resolution of $\lesssim 90 \mathrm{pc}$. The thermal fraction at $33 \mathrm{GHz}$ reaches unity at and around the peaks of each $\mathrm{H}$ in region; the spectral index between 33 and $94.5 \mathrm{GHz}$ additionally becomes both increasingly negative and positive away from the $\mathrm{H}$ iI region peaks, indicating an increase of non-thermal emission from diffusing cosmic-ray electrons and the possible presence of cold dust, respectively. While the ALMA observations were optimized for collecting continuum data, they also detected line emission from the $J=1 \rightarrow 0$ transitions of $\mathrm{HCN}$ and $\mathrm{HCO}^{+}$. The peaks of dense molecular gas traced by these two spectral lines are spatially offset from the peaks of the continuum emission for both extranuclear star-forming regions, indicating that our data reach an angular resolution at which one can spatially distinguish sites of recent star formation from the sites of future star formation. Finally, we find trends of decreasing dense gas fraction and velocity dispersion with increasing star formation efficiency among the regions observed, indicating that the dynamical state of the dense gas, rather than its abundance, plays a more significant role in the star formation process.
\end{abstract}

Key words: galaxies: individual (NGC 3627) - galaxies: star formation - H II regions - ISM: molecules radio continuum: general

\section{INTRODUCTION}

Stars form out of small pockets of dense molecular gas, with massive stars that are unambiguously young often embedded behind thick layers of dust. The dense star-forming gas is selectively traced by transitions with high critical densities, like the $J=1 \rightarrow 0$ transitions of $\mathrm{HCN}$ or $\mathrm{HCO}^{+}$(i.e., $n_{\text {crit }} \gtrsim 10^{5}$ $\mathrm{cm}^{-3}$ ). Likwise, recently formed stars are best studied by tracers such as free-free continuum at $\lambda \lesssim 1 \mathrm{~cm}$ or hydrogen recombination-line tracers that are robust against extinction and selectively sensitive to the massive young stars that live only a short time after their birth. To constrain the complete physical process of star formation, ideally both tracers would be observed on scales matched to individual star-forming regions. However, because of the faintness of both types of emission and the need for wide frequency coverage to isolate free-free emission from contaminants, this sort of study has been challenging before the current generation of radio and millimeter-wave telescopes, especially the Karl G. Jansky Very Large Array (VLA) and the Atacama Large Millimeter/ submillimeter Array (ALMA). In this paper, we combined new observations from ALMA and the VLA to report one of the first extragalactic cloud-scale comparisons of dense gas, traced by the high effective density transitions $\operatorname{HCN}(J=1 \rightarrow 0)$ and $\mathrm{HCO}^{+}(J=1 \rightarrow 0)$, and recent star formation traced by $\lambda \lesssim$ $1 \mathrm{~cm}$ free-free emission.
This paper represents the first results of a larger project to combine ALMA and the VLA to study continuum emission from nearby galaxies at frequencies ranging between $3-100 \mathrm{GHz}$. Emission at frequencies spanning $30-100 \mathrm{GHz}$ is expected to be dominated by free-free emission from $\mathrm{H}$ II regions, providing a highly robust measure of massive star formation activity unbiased by dust. Unfortunately, this emission component is energetically weak, making detections difficult and time consuming even for bright objects. To date, observations in this frequency range have been largely limited to Galactic H II regions (e.g., Mezger \& Henderson 1967), nearby dwarf irregular galaxies (e.g., Klein \& Graeve 1986), galaxy nuclei (e.g., Turner \& Ho 1983, 1994), nearby starbursts (e.g., Turner \& Ho 1985; Klein et al. 1988), and super star clusters within nearby blue compact dwarfs (e.g., Turner et al. 1998; Kobulnicky \& Johnson 1999). However, with ALMA now online, $3 \mathrm{~mm}$ bolometer arrays such as MUSTANG (Dicker et al. 2008), along with recent improvements to the backends of existing radio telescopes, such as the Caltech Continuum Backend on the Robert C. Byrd Green Bank Telescope and the Wideband Interferometric Digital ARchitecture correlator on the VLA, the availability of increased bandwidth is making it possible to conduct investigations for larger samples of extragalactic objects at frequencies $\gtrsim 30 \mathrm{GHz}$ (e.g., Murphy et al. 2011, 2012; Nikolic \& Bolton 2012). 
Table 1

Source Names and Imaging Summary

\begin{tabular}{|c|c|c|c|c|c|c|}
\hline Source & $\begin{array}{c}\text { R.A. } \\
(\mathrm{J} 2000)\end{array}$ & $\begin{array}{c}\text { Decl. } \\
(\mathrm{J} 2000)\end{array}$ & Beam $(33 \mathrm{GHz})$ & $\begin{array}{c}\sigma_{33 \mathrm{GHz}} \\
\left(\mu \mathrm{Jy} \mathrm{bm}^{-1}\right)\end{array}$ & Beam $(94.5 \mathrm{GHz})$ & $\begin{array}{c}\sigma_{94.5 \mathrm{GHz}} \\
\left(\mu \mathrm{Jy} \mathrm{bm}^{-1}\right)\end{array}$ \\
\hline NGC 3627 & 112015.0 & +125930 & $2 ! \prime 83 \times 1 " .83$ & 26 & $1 ! .89 \times 1 " .66$ & 32 \\
\hline NGC 3627 Enuc. 1 & 112016.2 & +112016 & $2 ! " 45 \times 2$ ". 03 & 20 & $1 "$ " $88 \times 1{ }^{\prime \prime} 67$ & 32 \\
\hline NGC 3627 Enuc. 2 & 112016.3 & +112016 & $2 ! .55 \times 2 ! .08$ & 25 & $1 ! .89 \times 1 " ! 67$ & 32 \\
\hline
\end{tabular}

Taking advantage of this new capability, the Star Formation in Radio Survey (SFRS; see Murphy et al. 2012), targets 118 star-forming regions (56 nuclear and 62 extranuclear) in 56 nearby galaxies $(d<30 \mathrm{Mpc})$ that had been observed at infrared and optical wavelengths as part of the SINGS (Kennicutt et al. 2003) and KINGFISH (Kennicutt et al. 2011) legacy programs. Of these, 112 (50 nuclei and 62 extranuclear regions) have $\delta>-35^{\circ}$ and are thus observable with the VLA, while 54 are observable with ALMA (i.e., $\delta<30^{\circ}$ ). We observed nine of these regions at frequencies near $95 \mathrm{GHz}$ using ALMA's Band 3 receiver during the second early science campaign ("Cycle 1"). All nine of these targets had already been observed by the VLA.

Thanks to ALMA's strong multiplexing capabilities and excellent sensitivity, we were also able to make a simultaneous search for bright line emission near $95 \mathrm{GHz}$. Specifically, the bandpass always covers the rest-frame $J=1 \rightarrow 0$ transition of $\mathrm{HCO}^{+}$and often includes the $J=1 \rightarrow 0$ transition of $\mathrm{HCN}$. These are two of the standard extragalactic tracers of dense molecular gas (Gao \& Solomon 2004; Graciá-Carpio et al. 2006). Thus our combined VLA and ALMA observations yield an unbiased view of the ionizing radiation emitted by massive young stars and a snapshot of the kind of bright, dense structures that might give birth to these regions.

In this paper, we report on the combination of VLA and ALMA data for three regions in the nearby galaxy NGC 3627 (M66), having a kinematic local standard of rest velocity $v_{\text {LSRK }}=726 \pm 3 \mathrm{~km} \mathrm{~s}^{-1}$. Along with NGC 3628 and NGC 3623, NGC 3627 makes up the well-known Leo Triplet galaxy group. It is classified with a morphological type of $\mathrm{SABb}$, located at a distance of $9.38 \pm 0.35 \mathrm{Mpc}$ (Freedman et al. 2001), and hosts a Seyfert 2 AGN (Ho et al. 1997; Moustakas et al. 2010). An $\mathrm{H}$ I plume extending $\approx 50^{\prime}$ indicates that the two largest spirals in the group, NGC 3627 and 3628, have interacted in the past (e.g., Haynes et al. 1979). NGC 3627 has comparable amounts of atomic and molecular gas (Helfer et al. 2003; Walter et al. 2008), which is a rather high molecular gas fraction compared to other local star-forming galaxies (e.g., Saintonge et al. 2011). It has been suggested that the high $\mathrm{H}_{2} / \mathrm{H}_{\mathrm{I}}$ mass ratio in NGC 3627 is the result of the tidal interaction with NGC 3628, since this galaxy has stripped much of the H I originally in NGC 3627 (Zhang et al. 1993).

The paper is organized as follow: In Section 2 we describe the data as well as the analysis procedures used in the present study. In Section 3 we present our results and discuss their implications. Our main conclusions are then summarized in Section 4.

\footnotetext{
${ }^{10}$ Kinematic LSR velocity and morphological type taken from the NASA Extragalactic Database (NED; http://nedwww.ipac.caltech.edu).
}

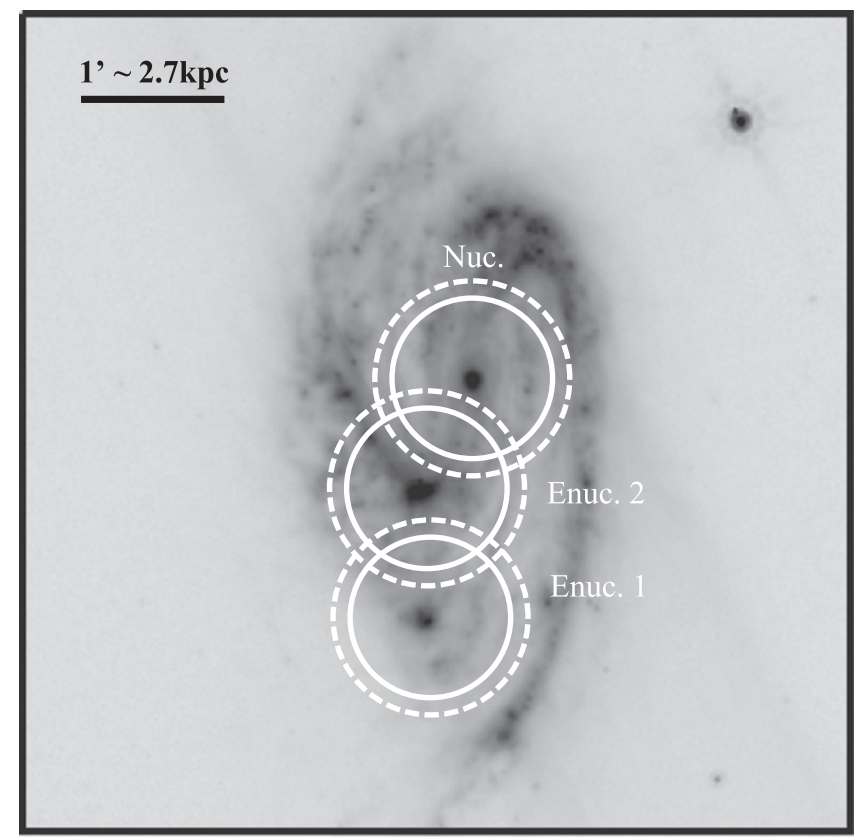

Figure 1. Locations of the VLA (dashed line) and ALMA (solid line) pointings for each region in NGC 3627 overlaid on a Spitzer $\lambda=8 \mu \mathrm{m}$ grayscale image. The circles outline the FWHMs of the VLA and ALMA primary beams at 33 and $94.5 \mathrm{GHz}$, respectively.

\section{DATA AND ANALYSIS}

We targeted the nucleus and two extranuclear star-forming regions in NGC 3627 with both ALMA and the VLA (Table 1), achieving a common resolution of $\approx 2^{\prime \prime}$, which is $\approx 90 \mathrm{pc}$ at the distance of NGC 3627. In Figure 1 we identify the locations of each region targeted in NGC 3627, showing the full width at half maximum (FWHM) of the VLA and ALMA primary beams at 33 and $94.5 \mathrm{GHz}$ on a Spitzer $\lambda=8 \mu \mathrm{m}$ grayscale image. The fields were selected to target individual massive star-forming regions, and the $\lambda=8 \mu \mathrm{m}$ image shows that each field is centered on a substantial concentration of bright polycyclic aromatic hydrocarbon emission, indicating a combination of intense radiation and abundant small dust grains. For the case of NGC 3627, this yields a sample that includes the nuclear disk, an isolated $\mathrm{H}$ II region (Enuc. 1), and the star-forming complex at the end of a bar (Enuc. 2), allowing us to compare star formation and molecular gas properties for three very different environmental conditions.

We worked with four data sets: VLA continuum observations at Ka band (29-37 GHz), ALMA continuum observations centered at $\approx 95 \mathrm{GHz}$, ALMA line observations of the $J=1 \rightarrow 0$ transition of $\mathrm{HCO}^{+}$and $\mathrm{HCN}$, and archival BIMA Survey of Nearby Galaxies (SONG; Helfer et al. 2003) line observations of the $J=1 \rightarrow 0$ transition of CO. 


\subsection{VLA Ka-band Data}

Details on all of the SFRS VLA Ka-band survey observations and data reduction can be found in E. J. Murphy et al. (2016, in preparation). Here, we present data only for NGC 3627. D-configuration observations were obtained in 2011 November (VLA/11B-032) and 2013 March (VLA/13A129). For the first round of observations, the 8-bit samplers were available, yielding $2 \mathrm{GHz}$ of simultaneous bandwidth, which we used to center $1 \mathrm{GHz}$ wide sub-bands at 32.5 and $33.5 \mathrm{GHz}$. For the latter run, the 3-bit samplers became available, yielding $8 \mathrm{GHz}$ of instantaneous bandwidth in $2 \mathrm{GHz}$ wide sub-bands centered at $30,32,34$, and $36 \mathrm{GHz}$. In both cases, 3C 286 was used as the flux density and bandpass calibrator, while J1118+1234 was used as the complex gain and telescope pointing calibrator. To reduce the VLA data, we used the Common Astronomy Software Applications (CASA; McMullin et al. 2007) and followed standard procedures.

\subsection{ALMA Band-3 Data}

In 2013 December as part of ALMA's Cycle 1 observing campaign, we obtained data for a subset of nine SFRS sources chosen to be sufficiently bright at $24 \mu \mathrm{m}$ and close enough on the sky that multiple sources could be observed in a single scheduling block. The array configuration was chosen to match the $\lesssim 2^{\prime \prime}$ synthesized beam of the VLA $33 \mathrm{GHz}$ data. While the goal of the ALMA observing program is to deliver continuum images at $\approx 95 \mathrm{GHz}$, we set the local oscillator frequency to $94.5 \mathrm{GHz}$, centering the four $1.875 \mathrm{GHz}$ wide spectral windows at $87.5,89.5,99.5$, and $100.5 \mathrm{GHz}$, to cover the rest frequencies of $\mathrm{HCN}(J=1 \rightarrow 0) / 88.6318 \mathrm{GHz}, \mathrm{HCO}^{+}(J=1 \rightarrow 0)$ /89.1885 GHz, HNC $(J=1 \rightarrow 0) / 90.6636 \mathrm{GHz}$, and $\mathrm{H} 40 \alpha$ / 99.0229 GHz. Like the VLA data, the ALMA data were reduced and calibrated using CASA following standard procedures as part of the ALMA quality assurance process. Here we present the nucleus and two extranuclear star-forming region in NGC 3627. The remaining ALMA sources, along with corresponding VLA data, will be presented together in a forthcoming paper.

\subsection{Archival CO $(J=1 \rightarrow 0)$ Data}

To investigate how the amount of dense star-forming gas compares to the total molecular gas reservoir, we make use of $J=1 \rightarrow 0$ CO data taken as part of the BIMA SONG survey (Helfer et al. 2003). The rms noise of the CO channel map is $41 \mathrm{mJy} \mathrm{bm}^{-1}$ in a $10 \mathrm{~km} \mathrm{~s}^{-1}$ channel. The resolution of the CO map is significantly coarser than our ALMA and VLA data, having a synthesized beam of 7 !" $3 \times 5$ !" 8 .

\subsection{Continuum Imaging and Photometry}

Calibrated VLA and ALMA measurement sets for each source were imaged using the task CLEAN in CASA. The Kaband images contain data from both sets of observations, but are heavily weighted by the $13 \mathrm{~A}$ semester observations as those include significantly more data. The mode of CLEAN was set to multifrequency synthesis (MFs; Conway et al. 1990; Sault \& Wieringa 1994). We chose to use Briggs weighting with ROBUST $=0.5$, and set the variable NTERMS $=2$, which allows the cleaning procedure to also model the spectral index variations on the sky. To help deconvolve extended low- intensity emission, we took advantage of the multiscale clean option (Cornwell 2008; Rau \& Cornwell 2011) in CASA, searching for structures with scales $\approx 1$ and 3 times the FWHM of the synthesized beam. A primary beam correction was applied using the CASA task IMPBCOR before analyzing the images. The primary-beam-corrected continuum images at 33 and $94.5 \mathrm{GHz}$ for each of the three targeted sources are shown in Figure 2. The synthesized beamwidths and rms noises of each image are given in Table 1. We also note that the largest angular scales that the ALMA and VLA images should be sensitive to are $\approx 25^{\prime \prime}$ and $44^{\prime \prime}$, respectively.

To measure the integrated flux densities and source sizes from each field, we used the task IMFIT in CASA to fit sources within a circular aperture having a radius of $15^{\prime \prime}$. In Table 2 we list the deconvolved source parameters from IMFIT, including the positions of the source components, peak brightnesses $\left(S_{\mathrm{P}}\right)$, integrated flux densities $\left(S_{\mathrm{I}}\right)$, deconvolved source sizes $\left(\theta_{\mathbf{M}} \times \theta_{\mathrm{m}}\right)$, and corresponding brightness temperatures $\left(T_{\mathrm{b}}\right)$. In addition to the errors reported by IMFIT, the uncertainties on the peak brightnesses and integrated flux densities include the contribution from image rms, as well as an absolute calibration uncertainty of 3\% at $33 \mathrm{GHz}$ (Perley \& Butler 2013) and 5\% at 94.5 GHz (ALMA Cycle 1 Technical Handbook), which in fact dominates the uncertainties. Clearly for the case of Enuc. 2, which is an elongated structure at the end of the bar, a singlecomponent fit is not the most appropriate.

\subsection{Line Imaging and Photometry}

We detected the $J=1 \rightarrow 0$ lines of $\mathrm{HCN}$ and $\mathrm{HCO}^{+}$ toward each targeted region in NGC 3627. We did not detect $\mathrm{H} 40 \alpha$ for any of the targeted regions, and our frequency coverage missed HNC $(J=1 \rightarrow 0)$ in each case. Similar to the continuum imaging, the line data were imaged using the task CLEAN in CASA. However, before the line images were created, the data were first continuum subtracted using the CASA task UVCONTSUB. The rms noise of the ALMA channel maps is $\approx 1.2 \mathrm{mJy} \mathrm{bm}^{-1}$ in a $10 \mathrm{~km} \mathrm{~s}^{-1}$ channel.

Moment 0 maps were constructed by integrating the spectra at each pixel of the spectral cubes. These are overlaid on the continuum maps in Figure 2. Unfortunately, the detection of HCN toward the nucleus is cut off by the bandpass at a radial velocity of $v_{\mathrm{LSRK}} \approx 800 \mathrm{~km} \mathrm{~s}^{-1}$. The moment 0 map for $\mathrm{HCO}^{+}$toward the nucleus shows that we are missing higher velocity $\mathrm{HCN}$ emission to the north. This can also be seen in Figure 3, which shows the spectra of both $\mathrm{HCN}$ and $\mathrm{HCO}^{+}$for all targeted regions. The spectra were extracted using a circular aperture centered on the $94.5 \mathrm{GHz}$ source positions returned by IMFIT having a diameter that was $20 \%$ larger than the corresponding source size major axis convolved with the $94.5 \mathrm{GHz}$ beam. We therefore exclude the nuclear HCN line emission in this study. For all other regions in Figure 3, the $\mathrm{HCN}$ and $\mathrm{HCO}^{+}$line profiles are fit by a Gaussian. The corresponding velocity dispersions are given the each panel, along with uncertainties that were estimated using the errors per channel along with a Monte Carlo approach.

Given that the CO $(J=1 \rightarrow 0)$ image has a much coarser angular resolution than our ALMA line images, we must first convolve our images to the resolution of the $\mathrm{CO}$ map before estimating dense molecular gas fractions. This must similarly be done for the continuum ( 33 and $94.5 \mathrm{GHz}$ ) data for estimating star formation efficiencies (SFE; see Section 3.3). 

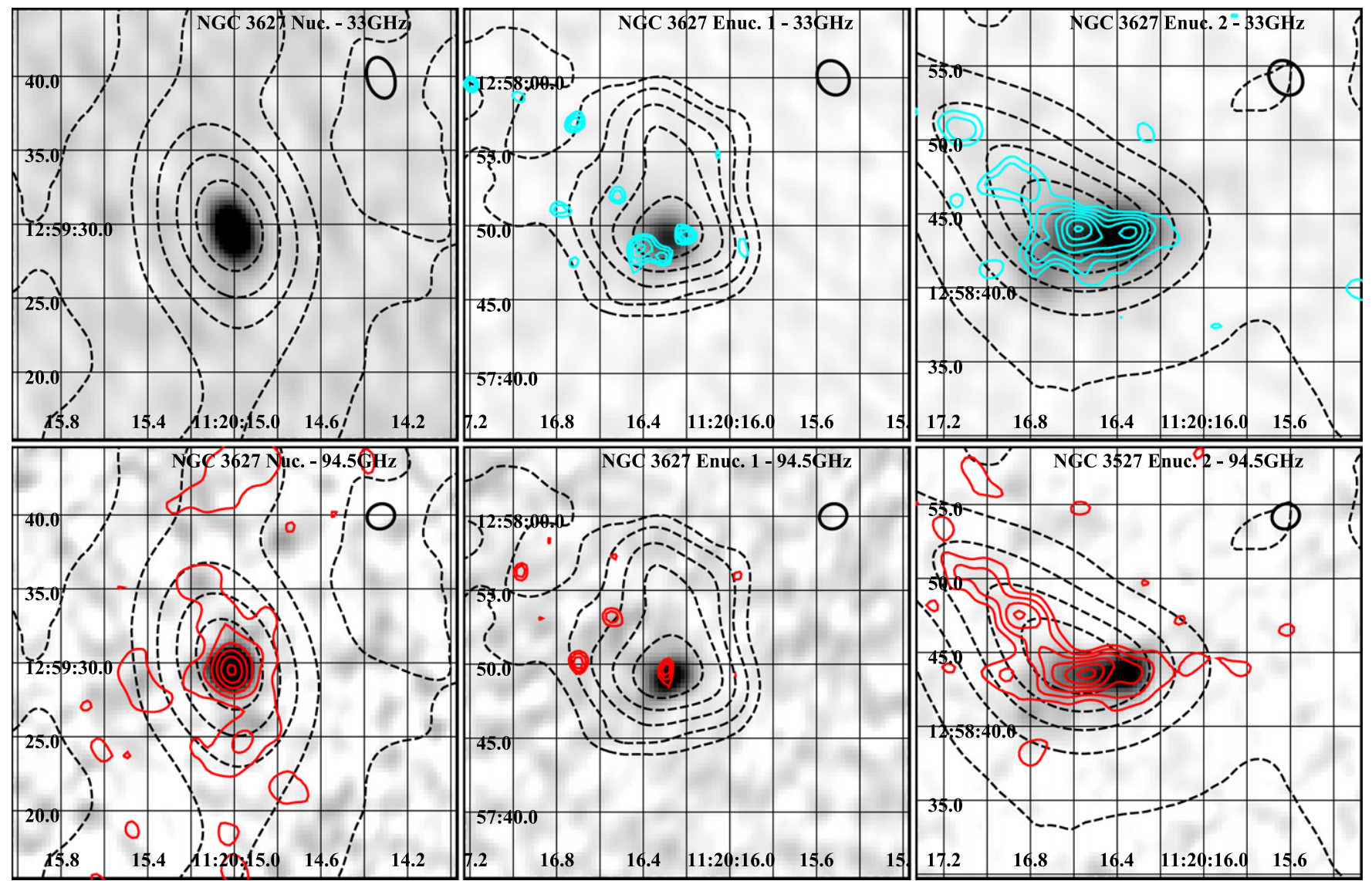

Figure 2. Continuum images of the three regions at 33 and $94.5 \mathrm{GHz}$ are shown in the first and second rows, respectively. Coordinates are in J2000. The restoring beam size (FWHM) is illustrated in the upper right corner of each panel. At the distance of NGC $3627,1^{\prime \prime}$ linearly projects to $\approx 45.5 \mathrm{pc}$ on the sky. CO contours are overlaid on each grayscale image as black dashed lines. The resolution of the CO images is far lower than in our ALMA or VLA images, being 7 .' $3 \times 5$ !' 8 . HCN contours (cyan) are overlaid on the $33 \mathrm{GHz}$ grayscale images in the top three panels, while $\mathrm{HCO}^{+}$contours (red) are overlaid on the $94.5 \mathrm{GHz}$ grayscale images in the bottom three panels. In all cases, contour lines are drawn using a linear scaling starting from the $2 \sigma$ value. As discussed in the text, the full velocity range of HCN emission toward the nucleus is cutoff by the bandpass at $v_{\text {LSRK }} \approx 800 \mathrm{~km} \mathrm{~s}^{-1}$, and therefore not shown.

Table 2

VLA and ALMA Continuum Properties

\begin{tabular}{|c|c|c|c|c|c|c|}
\hline Source & $\begin{array}{l}\text { R.A. } \\
(\mathrm{J} 2000)\end{array}$ & $\begin{array}{l}\text { Decl. } \\
\text { (J2000) }\end{array}$ & $\begin{array}{c}S_{\mathrm{P}} \\
\left(\mathrm{mJy} \mathrm{bm}^{-1}\right)\end{array}$ & $\begin{array}{c}S_{\mathrm{I}} \\
(\mathrm{mJy})\end{array}$ & $\theta_{\mathrm{M}} \times \theta_{\mathrm{m}}$ & $\begin{array}{l}T_{\mathrm{b}} \\
(\mathrm{K})\end{array}$ \\
\hline \multicolumn{7}{|c|}{$33 \mathrm{GHz}$ Components } \\
\hline NGC 3627 Enuc. 1 & 112016.30 & +125749.1 & $0.77 \pm 0.03$ & $1.66 \pm 0.09$ & 2 "! $52 \pm 0$ " $23 \times 2$ " $25 \pm 0 ! 27$ & $0.33 \pm 0.05$ \\
\hline NGC 3627 Enuc. 2 & 112016.46 & +125843.5 & $0.98 \pm 0.04$ & $5.35 \pm 0.27$ & $6 " .95 \pm 0$ ". $30 \times 3$ " $12 \pm 0$ " 17 & $0.28 \pm 0.02$ \\
\hline NGC 3627 Enuc. 1 & 112016.29 & +125749.2 & $0.63 \pm 0.05$ & $1.13 \pm 0.11$ & $1 "$ "95 \pm 0 "! $24 \times 1$ "' $24 \pm 0$ "! 22 & $0.063 \pm 0.015$ \\
\hline NGC 3627 Enuc. 2 & 112016.44 & +125843.5 & $0.68 \pm 0.05$ & $3.23 \pm 0.25$ & $5 "$ " $66 \pm 0$ ". $36 \times 1$ " $85 \pm 0$ ". 15 & $0.042 \pm 0.005$ \\
\hline
\end{tabular}

The resolution matching was carried out using the task IмSмоотн in CASA, before re-gridding each image to a common pixel scale. For such measurements requiring the convolved data, photometry was carried out by simply taking the peak brightness within a circular aperture having a radius of $15^{\prime \prime}$ (see Table 3), as this provides a way to mitigate any differences in our comparisons that may arise from being observed with different interferometers having different sensitivities on different spatial scales.

\section{RESULTS AND DISCUSSION}

In the following section we present our results along with a brief discussion about their implications for the star formation activity in the three regions observed in NGC 3627.

\subsection{Spectral Indices and Thermal Radio Fractions}

We created a 33-to-94.5 GHz spectral-index map (i.e., $\alpha$, where $S_{\nu} \propto \nu^{\alpha}$ ) of each source after convolving the slightly 


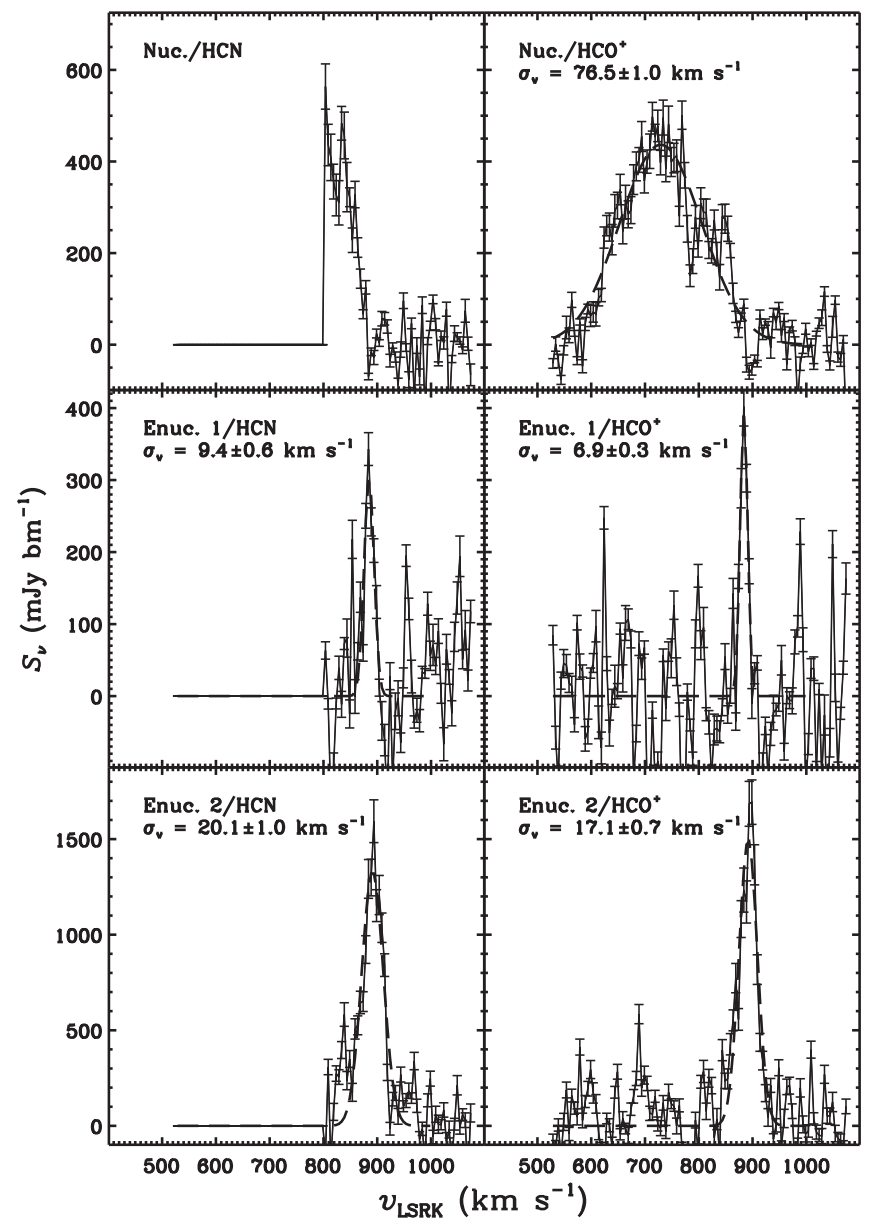

Figure 3. $\mathrm{HCN}$ and $\mathrm{HCO}^{+}$spectra $\left(5 \mathrm{~km} \mathrm{~s}^{-1}\right.$ channels) for each of the three targeted regions within NGC 3627 extracted using a circular aperture centered on the $94.5 \mathrm{GHz}$ source positions returned by IMFIT. The diameter of each aperture was $20 \%$ larger than the corresponding source size major axis convolved with the $94.5 \mathrm{GHz}$ beam. The full velocity range of $\mathrm{HCN}$ emission toward the nucleus is cutoff by the bandpass at $v_{\mathrm{LSRK}} \approx 800 \mathrm{~km} \mathrm{~s}^{-1}$. For all other regions, the spectral lines are fit by Gaussians (dashed lines), and the corresponding fitted velocity dispersions and uncertainties are given in the upper left corner of each panel.

higher resolution $94.5 \mathrm{GHz}$ data to match the beam of the $33 \mathrm{GHz}$ data and putting the data on the same grid. In the top panels of Figure 4 the spectral indices are overlaid on $94.5 \mathrm{GHz}$ continuum images for which pixels not detected at the $3 \sigma$ level have been clipped. Focusing only on the regions (i.e., pixels) detected at $>3 \sigma$ in each map, we measured mean spectral indices of $\alpha_{33 \mathrm{GHz}}^{94.5 \mathrm{GHz}}=-0.10,-0.15$, and -0.27 for the nucleus and extranuclear regions 1 and 2, respectively. The corresponding standard deviations over these regions are $0.20,0.18$, and 0.15 , respectively. As expected, these are relatively flat, being $<-0.5$ for each discrete source. We note that the spectral indices in some cases become positive in the outskirts of the $\mathrm{H}$ II regions, which may indicate a significant contribution of thermal dust to the $94.5 \mathrm{GHz}$ emission, although the uncertainties on the spectral indices in the $\mathrm{H}$ II region outskirts become large.

If we assume a fixed non-thermal spectral index for each source, we can use the measured spectral indices to estimate the fractional contributions from thermal emission (e.g., Klein et al. 1984; Murphy et al. 2012). While most sensitive to the assumption of the non-thermal spectral index, this simple thermal decomposition also assumes that the free-free emission does not become optically thick at $\nu \gtrsim 30 \mathrm{GHz}$ (e.g., Murphy et al. 2010b), and that there is an insignificant contribution of both anomalous microwave emission at $\sim 33 \mathrm{GHz}$ (e.g., Murphy et al. 2010a) and thermal dust emission at $\sim 94.5 \mathrm{GHz}$. As discussed below, the few regions where there may be evidence for thermal dust emission are excluded from the thermal fraction calculations. We took the non-thermal spectral index to be $\alpha^{\mathrm{NT}}=-0.85$, which is the average nonthermal spectral index found among the 10 star-forming regions studied in NGC 6946 by Murphy et al. (2011) and very similar to the average value found by Niklas et al. (1997, i.e., $\alpha^{\mathrm{NT}}=-0.83$ with a scatter of $\sigma_{\alpha^{\mathrm{NT}}}=0.13$ ) globally for a sample of 74 nearby galaxies. The $33 \mathrm{GHz}$ thermal fraction contours are overlaid on the $33 \mathrm{GHz}$ continuum images in the bottom panels of Figure 4, indicating thermal fractions near unity on the peaks of the continuum emission.

While NGC 3627 is known to host an AGN, the AGN does not appear to contribute significantly to the radio continuum emission. Filho et al. (2004) reported a $5 \mathrm{GHz}$ flux density of $<0.3 \mathrm{mJy}$ within a 3.6 mas $\times 1.4$ mas beam using the VLBA. Scaling this to $33 \mathrm{GHz}$ assuming a non-thermal spectral index of $\alpha^{\mathrm{NT}}=-0.85$ results in an estimated AGN contribution to the $33 \mathrm{GHz}$ emission of $<60 \mu \mathrm{Jy}$, or $<3 \%$ of the integrated $33 \mathrm{GHz}$ emission for the nuclear component reported by IMFIT (see Section 2.4). We therefore assume that the nuclear emission is primarily powered by star formation in the rest of the analysis.

Ignoring those regions for which the spectral index was measured to be flatter than -0.1 (i.e., the spectral index for pure free-free emission) by more than $1 \sigma$, where there may be a contribution from thermal dust, we measure corresponding mean $33 \mathrm{GHz}$ thermal fractions over the nucleus, Enuc. 1 and 2 of $85 \%, 82 \%$ and $70 \%$, respectively. The corresponding standard deviations measured over these regions are $19 \%$, $19 \%$ and $20 \%$. The thermal fraction distribution and average values are in agreement with the results presented by Murphy et al. (2012), who reported an average $33 \mathrm{GHz}$ thermal fraction of $\approx 76 \%$ with a dispersion of $24 \%$ for their entire sample, and even higher values (i.e., $>90 \%$, on average) for all sources resolved on scales of $\lesssim 500 \mathrm{pc}$. We note that assuming a much flatter non-thermal spectral index has little effect on the estimated thermal fraction given the flat spectral indices measured between 33 and $94.5 \mathrm{GHz}$. For example, by instead assuming $\alpha^{\mathrm{NT}}=-0.6$, the mean $33 \mathrm{GHz}$ thermal fractions over the nucleus, Enuc. 1, and 2 are $80 \%, 76 \%$, and $62 \%$, respectively, which are well within the quoted standard deviations.

\subsection{Spatial Offsets between Continuum and Line Emission}

One of the most obvious results from an inspection of the maps in Figure 2 is the spatial offset between the peaks of the continuum and line emission. This is especially evident for Enuc. 2 (i.e., the bar end) for which both dominant $\mathrm{HCN}$ and $\mathrm{HCO}^{+}$peaks are well to the east of the 33 and $94.5 \mathrm{GHz}$ continuum peaks. The continuum and molecular gas are spatially offset in the plane of the sky by $\approx 3^{\prime \prime}(\approx 130$ pc at the distance of NGC 3627).

The critical density of both the $J=1 \rightarrow 0$ lines of $\mathrm{HCN}$ and $\mathrm{HCO}^{+}$is $n_{\text {crit }} \gtrsim 10^{5} \mathrm{~cm}^{-3}$, and thus probes the densest, UVshielded gas that is likely in the process of, or soon will be, forming stars. Assuming that the 33 and $94.5 \mathrm{GHz}$ continuum 
Table 3

Photometry Incorporating CO Data

\begin{tabular}{|c|c|c|c|c|}
\hline Source & $\begin{array}{c}I_{\mathrm{CO}} \\
\left(\mathrm{K} \mathrm{Km} \mathrm{s}^{-1}\right)\end{array}$ & $\begin{array}{c}I_{\mathrm{HCN}} \\
\left(\mathrm{K} \mathrm{Km} \mathrm{s}^{-1}\right)\end{array}$ & $\begin{array}{c}I_{\mathrm{HCO}^{+}} \\
\left(\mathrm{K} \mathrm{Km} \mathrm{s}^{-1}\right)\end{array}$ & $\begin{array}{c}S_{33 \mathrm{GHz}} \\
\left(\mathrm{mJy} \mathrm{bm}^{-1}\right)\end{array}$ \\
\hline NGC 3627 & $375.47 \pm 19.3$ & $4.54 \pm 0.48$ & $13.80 \pm 0.81$ & $2.10 \pm 0.06$ \\
\hline NGC 3627 Enuc. 1 & $48.19 \pm 4.4$ & $1.05 \pm 0.23$ & $0.50 \pm 0.20$ & $1.56 \pm 0.05$ \\
\hline
\end{tabular}

Note. Photometry was carried out after convolving both VLA and ALMA maps to the 7 "! $3 \times 5$ "! 8 sythesized beam of the BIMA SONG $J=1 \rightarrow 0$ CO data.

is primarily powered by free-free emission, as suggested by the results presented in Section 3.1, and thus robustly traces ongoing star formation that is $\lesssim 10$ Myr old, this offset suggests that we have reached the spatial resolution at which we can reliably separate the fuel stockpiles from the current generation of star formation.

Pan et al. (2013) similarly report $\sim 100$ pc offsets between peaks of dense gas $(\mathrm{HCN} J=1 \rightarrow 0)$ and star formation $(3 \mathrm{~cm}$ radio continuum) in the starburst ring of the barred galaxy NGC 7522. These authors suggest that dense-gas formation is promoted by gas cloud collisions at the intersections between the galaxy bar and ring orbits, and that offsets between the dense gas peaks and those of ongoing star formation occur farther downstream from the orbit contact points. Similar conclusions have also been found for nuclear bars in nearby starbursts (e.g., Meier et al. 2008). Like NGC 7522, NGC 3627 is a barred galaxy with an inner molecular ring (Regan et al. 2002; Casasola et al. 2011, 2015), suggesting that similar dynamics may also be driving the formation of dense gas and its observed spatial offsets from the peaks of ongoing star formation activity in Enuc. 2.

Assuming this scenario to be true, we can make a rough estimate of the propagation time between the newly formed stars, as traced by the free-free radio continuum emission, and the dense gas peaks. The rotation speed of the NGC 3627's disk at the distance to the bar end (i.e., $\approx 100^{\prime \prime} \approx 4.5 \mathrm{kpc}$ ) is $\approx 204 \mathrm{~km} \mathrm{~s}^{-1}$ (de Blok et al. 2008). The bar pattern speed for NGC 3627 is $\approx 55 \mathrm{~km} \mathrm{~s}^{-1} \mathrm{kpc}^{-1}$ (Sheth et al. 2002), corresponding to $\approx 247 \mathrm{~km} \mathrm{~s}^{-1}$ at this distance, yielding a net velocity of $\approx 43 \mathrm{~km} \mathrm{~s}^{-1}$ between the disk and the bar. Thus, the propagation time is $\approx 3 \mathrm{Myr}$, less than the age of typical OB stars.

Another striking result from comparing the Enuc. 1 maps in Figure 2 is that the $\mathrm{HCN}$ and $\mathrm{HCO}^{+}$peaks are not co-spatial. The peak of the $\mathrm{HCO}^{+}$emission is located near the peak of the 33 and $94.5 \mathrm{GHz}$ continuum emission. However, the $\mathrm{HCN}$ emission peaks in regions that surround the $\mathrm{HCO}^{+}$and continuum peaks. This may be the result in variations in the (dense) gas density, given that the critical density of $\mathrm{HCN}$ is nearly and order of magnitude larger than that of $\mathrm{HCO}^{+}$(e.g., Meier \& Turner 2012). Stockpiles of ultra dense gas are likely still present in the immediate vicinity surrounding the newly formed $\mathrm{H}$ II region. Although, excitation effects may also be in play (e.g., see Section 3.3).

\subsection{Dense Gas Fractions and SFE}

In the top panel of Figure 5 we plot the dense molecular gas fraction against the estimated SFE for each region. The dense molecular gas fraction is taken to be the mass ratio of dense molecular gas traced by the $J=1 \rightarrow 0$ transition of $\mathrm{HCO}^{+}$to the total molecular gas traced by the $J=1 \rightarrow 0$ transition of $\mathrm{CO}$. For this calculation we assume $\mathrm{HCO}^{+}$-to- $\mathrm{H}_{2}$ and $\mathrm{CO}$-to-
$\mathrm{H}_{2}$ conversion factors of $\alpha_{\mathrm{HCO}^{+}} \approx 10 M_{\odot}\left(\mathrm{K} \mathrm{km} \mathrm{s}^{-1} \mathrm{pc}^{2}\right)^{-1}$ (e.g., Gao \& Solomon 2004, we assume $\alpha_{\mathrm{HCO}^{+}} \approx \alpha_{\mathrm{HCN}}$ ) and $\alpha_{\mathrm{CO}} \approx 4.3 \quad M_{\odot}\left(\mathrm{K} \mathrm{km} \mathrm{s}^{-1} \mathrm{pc}^{2}\right)^{-1}$ (e.g., Solomon \& Vanden Bout 2005; Bolatto et al. 2013), respectively. We only make use of the $\mathrm{HCO}^{+}$for deriving the dense molecular gas masses as we have data for all regions. The SFE, whose inverse is often referred to as the gas depletion time, is taken to be the ratio of the star formation rate (SFR) to the total molecular gas mass traced by the $J=1 \rightarrow 0$ transition of $\mathrm{CO}$. We converted the $33 \mathrm{GHz}$ spectral luminosity into a SFR using the calibration for pure thermal emission given in (Murphy et al. 2011, 2012) and assumed an average $33 \mathrm{GHz}$ thermal fraction of $79 \%$ (see Section 3.1). A clear trend of decreasing dense gas fraction with increasing SFE is observed. More specifically, the nuclear disk of NGC 3627 appears to have the largest fraction of its molecular gas in a dense phase, followed by the star-forming region at the end of the bar (Enuc. 2), and the isolated H II region (Enuc. 1). However, Enuc. 1 (the isolated $\mathrm{H}_{\text {II }}$ region), appears to be converting its molecular gas into stars more efficiently (i.e., $\approx$ a factor of 3 more rapidly) than either the star formation region at the end of the bar or the nuclear disk. By plotting the $\mathrm{HCO}^{+}$velocity dispersions from the fitted line profiles in Figure 3 against SFE in the middle panel of Figure 5, we find that the SFE for each region increases with decreasing velocity dispersion. Thus, perhaps unsurprisingly, the dynamical state of the dense gas appears to have a larger impact on the star formation process than the actual fraction of dense gas that is available for star formation.

Comparing the dense gas fraction and the efficiency of star formation on much larger scales, Usero et al. (2015) recently found systematic trends in both the dense gas fraction and the efficiency with which dense gas forms stars. That study included NGC 3627, though with a beam area two orders of magnitude larger than our study. Similar to our results, but using $\mathrm{HCN}$, they found that in $\approx 30$ disk galaxies, the apparent dense gas fraction increases by moving from the outer, low surface density parts of galaxies to the inner regions. At the same time, they found the apparent efficiency with which dense gas forms stars (for them, SFR/HCN) appears to decrease as one moves from the lower surface density disk to the inner parts of galaxies. They showed that these results could be explained by models of turbulent clouds (e.g., Krumholz \& McKee 2005; Federrath \& Klessen 2012) in which the average density and the turbulent Mach number in a cloud affect both the dense gas fraction and the ability of gas at different densities to form stars. Similar results have been found considering the apparently low rate at which dense gas forms stars in the central part of the Milky Way (e.g., Kauffmann et al. 2013; Longmore et al. 2013; Rathborne et al. 2014). With the improved resolution offered by ALMA, we are able to measure a velocity dispersion that may be more directly related to the turbulent velocity dispersion (at least outside the central 


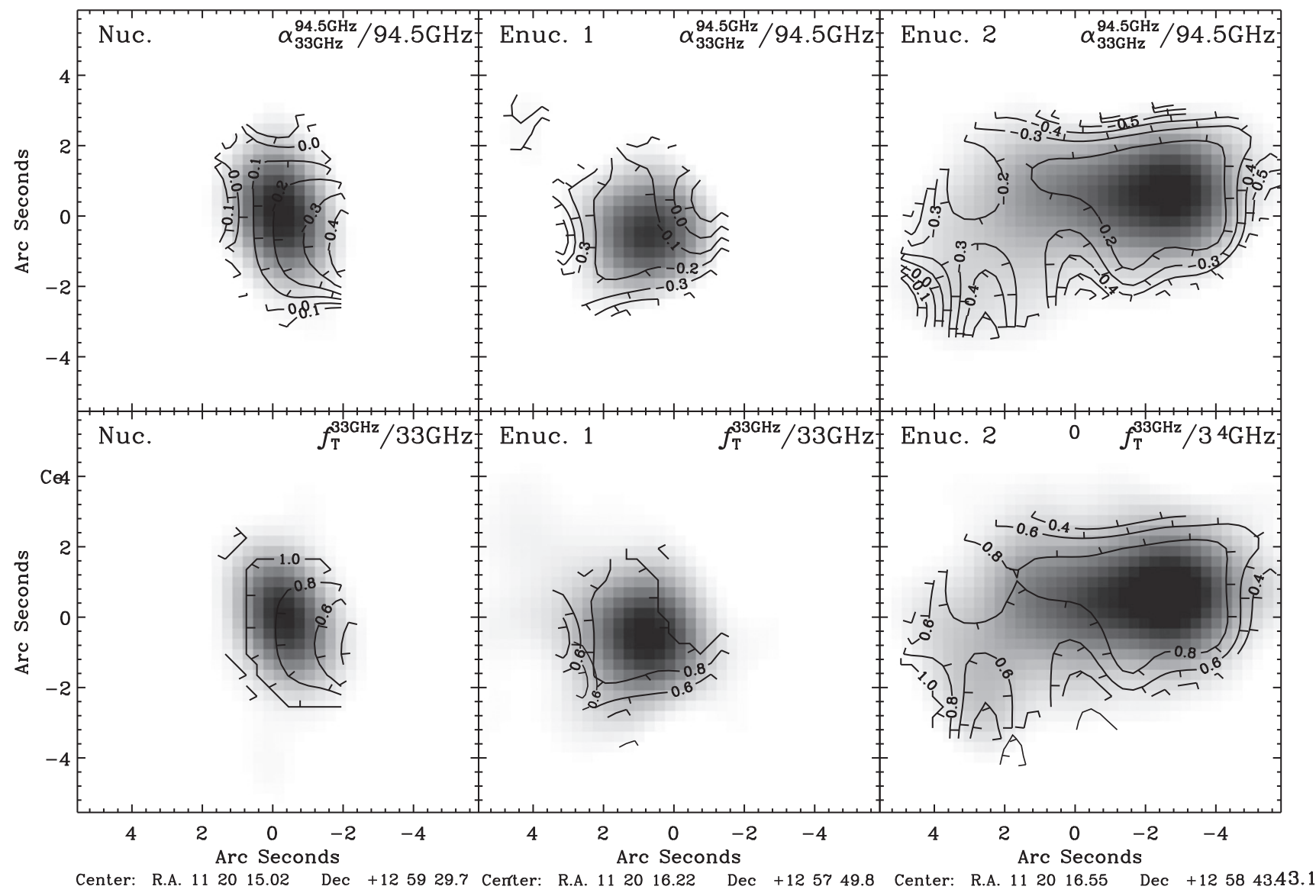

Figure 4. Top row: spectral index contours between 33 and $94.5 \mathrm{GHz}$ overlaid on the $94.5 \mathrm{GHz}$ continuum image of the nucleus (left), Enuc. 1 (middle), and Enuc. 2 (right). In each case, the spectral indices flatten at the peaks of the continuum emitting regions. Bottom row: estimated thermal fractions at $33 \mathrm{GHz}$ overlaid on the $33 \mathrm{GHz}$ continuum image of the nucleus (left), Enuc. 1 (middle), and Enuc. 2 (right) of NGC 3627. For both extranuclear star-forming regions, the $33 \mathrm{GHz}$ thermal fractions approach unity on the peaks of the continuum emitting regions.

region). The observation of variable dense gas efficiency $\left(\mathrm{SFR} / \mathrm{HCO}^{+}\right)$and suppressed star formation in the central, high dispersion part of the galaxy appears to agree at least qualitatively with these results.

Since we have relied on only the $J=1 \rightarrow 0$ transition of $\mathrm{HCO}^{+}$to derive the dense gas mass, and have assumed similar conversion factors for $\mathrm{HCN}$ and $\mathrm{HCO}^{+}$to dense $\mathrm{H}_{2}$, it is illustrative to see how the $\mathrm{HCN}$-to- $\mathrm{HCO}^{+}$line brightness ratio varies among regions. This is shown in the bottom panel of Figure 5, and plotted against SFE. The $\mathrm{HCN}$-to- $\mathrm{HCO}^{+}$line brightness ratio changes by a factor of $\approx 2$ between both extranuclear star-forming regions over a similar change in SFE. This trend may be due to gas excitation effects given that the abscissa is a measure of the number of ionizing photons (free electrons) per unit molecular gas mass. For instance, as discussed in Papadopoulos (2007), the $\mathrm{HCO}^{+}$abundance is known to be sensitive to the ionization degree of molecular gas, which can significantly reduce the $\mathrm{HCO}^{+}$abundance in starforming and highly turbulent molecular gas, while $\mathrm{HCN}$ remains abundant. Given the observed trend, this appears to be a plausible explanation. However, there are many alternative explanations for changes in the ratio of $\mathrm{HCN}$-to- $\mathrm{HCO}^{+}$line brightness ratio including variations in gas density (see, e.g., Meier \& Turner 2012). Given the large error bar on the linebrightness ratio for Enuc. 1, further speculation here is unwarranted. Regardless, it is worth pointing out that even if one ignores the large error bar, the trend observed in the top panel of Figure 5 would persist even if we instead used the $\mathrm{HCN}$-derived dense gas mass.

\section{CONCLUSIONS}

In this paper we have combined ALMA/Band-3 line and continuum images with VLA/Ka-band images to characterize the star formation activity on $\approx 100 \mathrm{pc}$ scales around three distinct regions within the nearby galaxy NGC 3627; i.e., a nuclear disk hosting an AGN, an isolated $\mathrm{H}$ II region (Enuc. 1), and a star-forming complex at the end of a bar (Enuc. 2). Our conclusions can be summarized as follows.

1. The thermal fraction at $33 \mathrm{GHz}$ is nearly unity at the peaks of the $\mathrm{H}$ II regions as mapped on $\approx 100 \mathrm{pc}$ scales, with an average value of $\approx 76 \%$ for both extranuclear star-forming regions. The mean thermal fraction at $33 \mathrm{GHz}$ among the three regions studied is $79 \%$ with a dispersion of $19 \%$. We additionally found the radio spectral index to become both increasingly negative and positive away from the peaks of the H II regions, indicating an increase of extended non-thermal emission from diffusing cosmic-ray electrons and the possible presence of cold dust, respectively.

2. The peaks of the $J=1 \rightarrow 0 \mathrm{HCN}$ and $\mathrm{HCO}^{+}$line emission are spatially offset from the peaks of the 33 and 


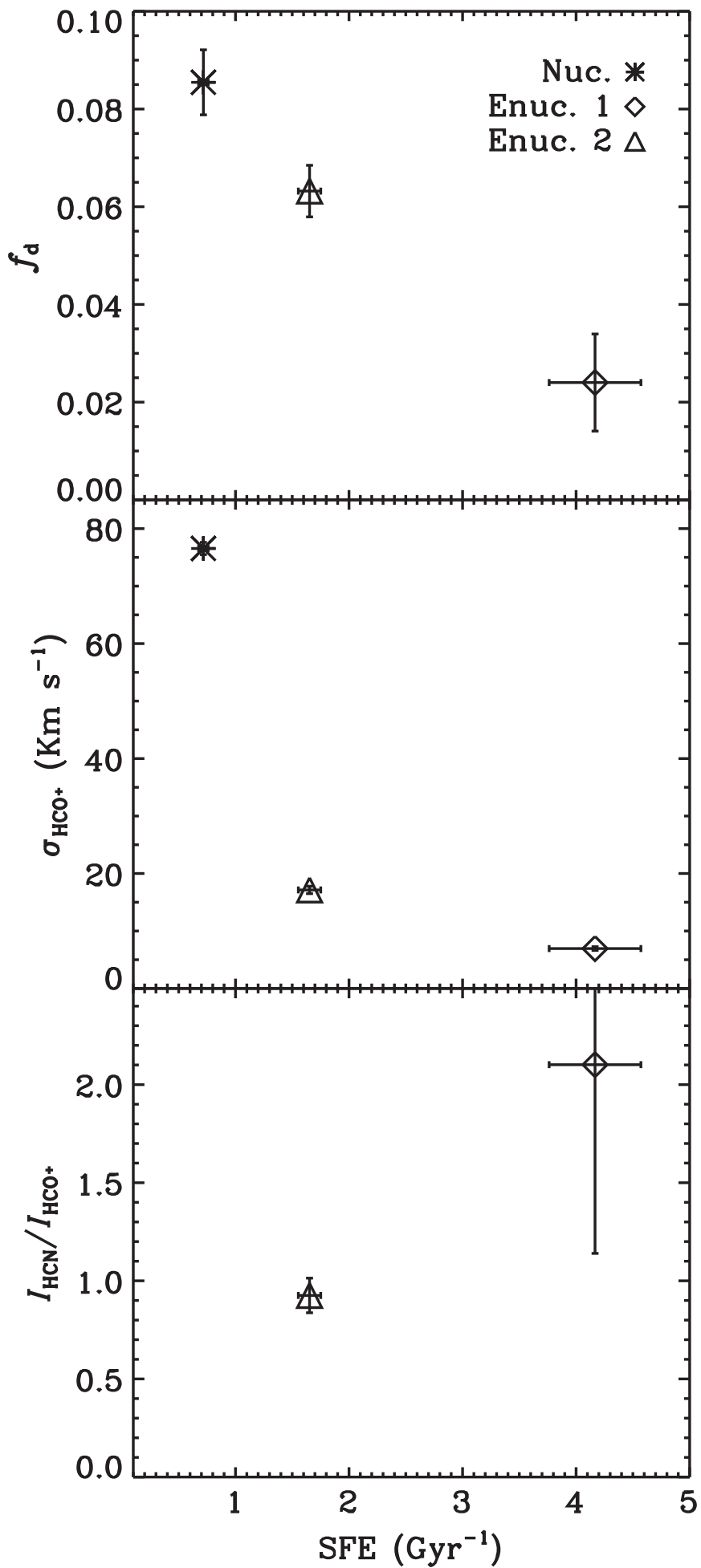

Figure 5. Top: dense gas fraction $f_{\mathrm{d}}$ plotted as a function of star formation efficiency for each region observed in NGC 3627. The quantities on the two axes are both normalized by $\mathrm{CO}$ luminosity, so some spurious correlation is expected in the presence of noise. However, to the contrary we observe an anticorrelation between star formation efficiency and dense gas fraction, which is opposite the sense expected from only noisy $\mathrm{CO}$ measurements. Middle: $\mathrm{HCO}^{+}$velocity dispersions from the fitted line profiles shown in Figure 3 plotted as a function of star formation efficiency for each region observed in NGC 3627. Bottom: ratio of $\mathrm{HCN}$ to $\mathrm{HCO}^{+}$brightnesses plotted against star formation efficiency, indicating a trend of increasing line brightness ratio with increasing measure of ionizing photons per unit molecular gas mass. Given that we are missing higher velocity $\mathrm{HCN}$ emission to the north of the nucleus, this data point is omitted in the bottom panel.
94.5 GHz continuum emission. For Enuc. 2, the continuum and gas are spatially offset in the plane of the sky by $\approx 3^{\prime \prime}(\approx 130 \mathrm{pc}$ at the distance of NGC 3627). Assuming that the 33 and $94.5 \mathrm{GHz}$ continuum is primarily powered by free-free emission, and that the $J=1 \rightarrow 0$ $\mathrm{HCN}$ and $\mathrm{HCO}^{+}$line emission is tracing dense, UVshielded gas, this indicates that our data reach an angular resolution at which one can spatially distinguish sites of current star formation from the fuel stockpiles for the next-generation of star formation.

3. Combining our ALMA and VLA observations with archival BIMA CO $(J=1 \rightarrow 0)$ data, we calculate dense gas fractions and SFE for each region, finding that the dense gas fraction decreases with increasing SFE. This suggests that an increase in the dense gas content of starforming regions does not reflect an increased efficiency for which parts of galaxies can turn molecular gas into stars among the three, diverse regions studied here. Specifically, Enuc. 1 (an isolated $\mathrm{H}$ II region) appears to be converting molecular gas into stars more efficiently than both the star-forming region at the end of the bar or the nuclear disk. We additionally find that the velocity dispersion of the dense gas in each region decreases with increasing SFE, indicating that the dynamical state of the dense gas, rather than its abundance, plays a larger role affecting ongoing star formation activity.

4. The $J=1 \rightarrow 0 \mathrm{HCN}$-to- $\mathrm{HCO}^{+}$line brightness ratio and SFE both vary by a factor of $\approx 2$ between the extranuclear $\mathrm{H}$ II regions. We speculate that this may be due to an increase in the ionization degree of molecular gas for the star-forming region having a higher SFE, which in turn reduces the $\mathrm{HCO}^{+}$abundance while $\mathrm{HCN}$ remains abundant. However, alternative explanations (e.g., variations in the gas density) may also be plausible.

We would like to thank the anonymous referee for useful comments that helped to improve the content and presentation of this paper. The National Radio Astronomy Observatory is a facility of the National Science Foundation operated under cooperative agreement by Associated Universities, Inc. This paper makes use of the following ALMA data: ADS/JAO. ALMA\#2012.1.00456.S. ALMA is a partnership of ESO (representing its member states), NSF (USA) and NINS (Japan), together with NRC (Canada) and NSC and ASIAA (Taiwan), in cooperation with the Republic of Chile. The Joint ALMA Observatory is operated by ESO, AUI/NRAO and NAOJ. This research has made use of the NASA/IPAC Extragalactic Database (NED), as well as the NASA/IPAC Infrared Science Archive, both of which are operated by the Jet Propulsion Laboratory, California Institute of Technology, under contract with the National Aeronautics and Space Administration.

\section{REFERENCES}

Bolatto, A. D., Wolfire, M., \& Leroy, A. K. 2013, ARA\&A, 51, 207

Casasola, V., Hunt, L., Combes, F., \& García-Burillo, S. 2015, A\&A, 577, A135

Casasola, V., Hunt, L. K., Combes, F., García-Burillo, S., \& Neri, R. 2011, A\&A, 527, A92

Conway, J. E., Cornwell, T. J., \& Wilkinson, P. N. 1990, MNRAS, 246, 490 Cornwell, T. J. 2008, ISTSP, 2, 793

de Blok, W. J. G., Walter, F., Brinks, E., et al. 2008, AJ, 136, 2648 
Dicker, S. R., Korngut, P. M., Mason, B. S., et al. 2008, Proc. SPIE, 7020, 702005

Federrath, C., \& Klessen, R. S. 2012, ApJ, 761, 156

Filho, M. E., Fraternali, F., Markoff, S., et al. 2004, A\&A, 418, 429

Freedman, W. L., Madore, B. F., Gibson, B. K., et al. 2001, ApJ, 553, 47

Gao, Y., \& Solomon, P. M. 2004, ApJS, 152, 63

Graciá-Carpio, J., García-Burillo, S., Planesas, P., \& Colina, L. 2006, ApJL, 640, L135

Haynes, M. P., Giovanelli, R., \& Roberts, M. S. 1979, ApJ, 229, 83

Helfer, T. T., Thornley, M. D., Regan, M. W., et al. 2003, ApJS, 145, 259

Ho, L. C., Filippenko, A. V., \& Sargent, W. L. W. 1997, ApJS, 112, 315

Kauffmann, J., Pillai, T., \& Goldsmith, P. F. 2013, ApJ, 779, 185

Kennicutt, R. C., Jr., Armus, L., Bendo, G., et al. 2003, PASP, 115, 928

Kennicutt, R. C., Calzetti, D., Aniano, G., et al. 2011, PASP, 123, 1347

Klein, U., \& Graeve, R. 1986, A\&A, 161, 155

Klein, U., Wielebinski, R., \& Beck, R. 1984, A\&A, 135, 213

Klein, U., Wielebinski, R., \& Morsi, H. W. 1988, A\&A, 190, 41

Kobulnicky, H. A., \& Johnson, K. E. 1999, ApJ, 527, 154

Krumholz, M. R., \& McKee, C. F. 2005, ApJ, 630, 250

Longmore, S. N., Bally, J., Testi, L., et al. 2013, MNRAS, 429, 987

McMullin, J. P., Waters, B., Schiebel, D., Young, W., \& Golap, K. 2007, in

ASP Conf. Ser. 376, Astronomical Data Analysis Software and Systems

XVI, ed. R. A. Shaw, F. Hill \& D. J. Bell (San Francisco, CA: ASP), 127 Meier, D. S., \& Turner, J. L. 2012, ApJ, 755, 104

Meier, D. S., Turner, J. L., \& Hurt, R. L. 2008, ApJ, 675, 281

Mezger, P. G., \& Henderson, A. P. 1967, ApJ, 147, 471
Moustakas, J., Kennicutt, R. C., Jr., Tremonti, C. A., et al. 2010, ApJS, 190,233

Murphy, E. J., Bremseth, J., Mason, B. S., et al. 2012, ApJ, 761, 97

Murphy, E. J., Condon, J. J., Schinnerer, E., et al. 2011, ApJ, 737, 67

Murphy, E. J., Helou, G., Condon, J. J., et al. 2010a, ApJL, 709, L108

Murphy, T., Cohen, M., Ekers, R. D., et al. 2010b, MNRAS, 405, 1560

Niklas, S., Klein, U., \& Wielebinski, R. 1997, A\&A, 322, 19

Nikolic, B., \& Bolton, R. 2012, MNRAS, 425, 1257

Pan, H.-A., Lim, J., Matsushita, S., Wong, T., \& Ryder, S. 2013, ApJ, 768, 57 Papadopoulos, P. P. 2007, ApJ, 656, 792

Perley, R. A., \& Butler, B. J. 2013, ApJS, 204, 19

Rathborne, J. M., Longmore, S. N., Jackson, J. M., et al. 2014, ApJL, 795, L25

Rau, U., \& Cornwell, T. J. 2011, A\&A, 532, A71

Regan, M. W., Sheth, K., Teuben, P. J., \& Vogel, S. N. 2002, ApJ, 574,126

Saintonge, A., Kauffmann, G., Kramer, C., et al. 2011, MNRAS, 415, 32

Sault, R. J., \& Wieringa, M. H. 1994, A\&AS, 108, 585

Sheth, K., Vogel, S. N., Regan, M. W., et al. 2002, AJ, 124, 2581

Solomon, P. M., \& Vanden Bout, P. A. 2005, ARA\&A, 43, 677

Turner, J. L., \& Ho, P. T. P. 1983, ApJL, 268, L79

Turner, J. L., \& Ho, P. T. P. 1985, ApJL, 299, L77

Turner, J. L., \& Ho, P. T. P. 1994, ApJ, 421, 122

Turner, J. L., Ho, P. T. P., \& Beck, S. C. 1998, AJ, 116, 1212

Usero, A., Leroy, A. K., Walter, F., et al. 2015, arXiv:1506.00703

Walter, F., Brinks, E., de Blok, W. J. G., et al. 2008, AJ, 136, 2563

Zhang, X., Wright, M., \& Alexander, P. 1993, ApJ, 418, 100 